\title{
ON THE EXISTENCE OF PERIODIC SOLUTIONS OF A CERTAIN CLASS OF SECOND ORDER NONLINEAR DIFFERENTIAL EQUATION
}

\author{
B. MEHRI AND D. SHADMAN
}

Abstract. A second order nonlinear differential equation is considered. Leray-Schauder principle is used to show the existence of periodic solutions. The results obtained are then applied to a specific example, where a computer program based on the fourth order Runge-Kutta method and Newton-Raphson algorithm is used to compute the periodic solutions.

Mathematics subject classification (1991): 34C25, 34A05.

Key words and phrases: Periodic solutions, differential equations.

\section{REFERENCES}

[I.] J. CRONIN, Fixed points and topological degree in nonlinear analysis, Math. Survey II, American Math. Soc., Providence, RI., 1964.

[II.] A. C. LAZER, On Schauder's fixed point theorem and forced second order nonlinear oscillations, J. Math. Anal. Appl. 21 (1968), 421-425.

[III.] R. REISSIG, On the existence of periodic solutions of certain non autonomous differential equations, Ann. Math. Pura Appl. 85 (1970), 230-240.

[IV.] Periodic Solution of a third order nonlinear differential equation, Annli di Mathematica 112 (1972), 193-198.

[V.] D. SHADMAN, B. MEHRI, On the periodic solutions of certain nonlinear third order differential equations, Zamm Z. angew. Math. Mech. 75, 2 (1995), 164-166.

[VI.] R. REISSIG, Perturbations of a certain critical $n$-th order differential equation, Bolletino della Unione Mathematica Italiana (4) II, Suppl. fase 3 (1975), 131-141.

[VII.] B. MeHrI, 203-210, Indian J. Pure Appl. Math. 21 (3) (1990).

[VIII.] H. O. TeJumola, Periodic Solutions of certain non-dissipative systems of third order differential equations, Colloquia Mathematica Societatis Janos Bolyai 30. Qualitative theory of differential equations, Szeged (Hungary) (1979).

[IX.] J. Stoer, R. BulRisch, Introduction to numerical Analysis, Springer Verlag, 1979. 